\author{
Jörgen M. P. Rovers \\ Elisabeth S. Louwerse \\ C. Peter C. de Jager
}

\section{Complete recovery from an unusual cause of coma}

Received: 30 November 2006

Accepted: 29 January 2007

Published online: 14 February 2007

(C) Springer-Verlag 2007

The Case Discussion format is intended to describe instructive cases in the field of intensive care medicine in a stepwise manner, allowing each step to be commented or discussed. This format might help to delineate the different steps of clinical reasoning in intensive care and highlight the important messages conveyed by the case

Keywords Coma - Acute disseminated encephalomyelitis

\section{Case}

A 51-year-old man was admitted to the emergency room because of progressive lethargy, slurred speech, weakness of the right arm and low-grade fever. Physical examination showed no additional neurological deficits. He had a history of a tick bite 6 months previously which had been treated with antibiotics. Apart from leukocytosis $\left(10.5 \times 10^{9} / \mathrm{l}\right)$ and raised C-reactive protein $(62 \mathrm{mg} / \mathrm{l})$, additional blood examination was normal. Cerebral contrast-enhanced computed tomography $(\mathrm{CT})$ revealed no abnormalities. Cerebrospinal fluid (CSF) examination showed an elevated cell count of $66 \times 10^{6}$ cells/ 1 (of which $97 \%$ were lymphocytes), slightly elevated protein concentration of $1.03 \mathrm{~g} / \mathrm{l}$, a normal glucose level of $3.7 \mathrm{mmol} / \mathrm{l}$ and an elevated pressure of $29 \mathrm{cmH}_{2} \mathrm{O}$.
Case discussion

The onset of progressive lethargy over a period of days, slurred speech and the weakness indicates a lesion of the central nervous system. Given the progression of lethargy, the fever and the absence of acute onset, an infectious origin seemed more likely. This was suggested by the CSF examination, showing an elevated cell count, especially lymphocytes. The differential diagnosis consisted of (viral) meningo-encephalitis most likely caused by enteroviruses, arboviruses or herpesviruses - neuroborelliosis and vasculitis. Antibiotics and antiviral drugs were started.

\section{Case}

Within 2 days, the patient's condition deteriorated into a deep coma requiring mechanical ventilation. Initial treatment consisted of acyclovir and ceftriaxone, anticipating a possible neuroborelliosis and herpes simplex encephalitis. However, repeated blood and CSF cultures were normal, making an infective origin less likely. Serological tests for Lyme disease and repeated polymerase chain reaction (PCR) testing for herpes simplex virus (HSV) were negative. Systemic vasculitis was ruled out. The patient remained in an areactive coma without focal signs and abnormal brainstem reflexes. An electro-encephalogram (EEG) after 1 week showed non-specific diffuse slow activity. Three weeks after admission cerebral gadoliniumenhanced magnetic resonance imaging (MRI) was performed, showing numerous hyperintense lesions, on T2-weighted images, of the brainstem, the left cerebellar hemisphere, the basal ganglia on both sides and in periventricular locations (Fig. 1a-c).
Case discussion

On ICU admission the main medical problems were (1) respiratory insufficiency requiring mechanical ventilation, (2) progressive loss of consciousness and (3) the unknown origin of the coma. Maintenance of an adequate airway and concomitant aspiration pneumonia necessitated mechanical ventilation. There are numerous possible causes of progressive loss of consciousness, including cerebrovascular accidents, cerebral infections (viral, bacterial, parasites, tuberculosis, Lyme disease), neoplastic and auto-immune diseases (sarcoidosis, vasculitis, SLE). Despite the elevated white cell count and the presence of protein in the CSF, no infectious origin was determined. Screening for HIV and coagulation disorders was negative. Repeated blood and CSF cultures were negative, and there was no response after 14 days of ceftriaxone and acyclovir. Nevertheless CSF analysis after treatment was interpreted cautiously. Many viruses responsible for meningo-encephalitis cannot be identified using common tests. MRI showed multiple hyperintense brain lesions, especially of the brainstem. Because of the massive involvement of the brainstem and persistence of coma for 2 weeks, the prognosis was considered poor.

\section{Case}

Acute disseminated encephalomyelitis (ADEM) could not be ruled out, so treatment was continued. Additionally, treatment with intravenous dexamethasone (4 mg four times a day) was started. Upon treatment with corticosteroids for 4 weeks the patient made a slow but full recovery within 2 months. After 2 months 
Fig. 1 a-c T2-weighted MRI of the brain, demonstrating hyperintense lesions of a brainstem and left cerebellar peduncle, b basal ganglia and c left periventricular region. c-e T2weighted MRI of the brain 2 months after therapy, demonstrating a decrease in white matter lesions

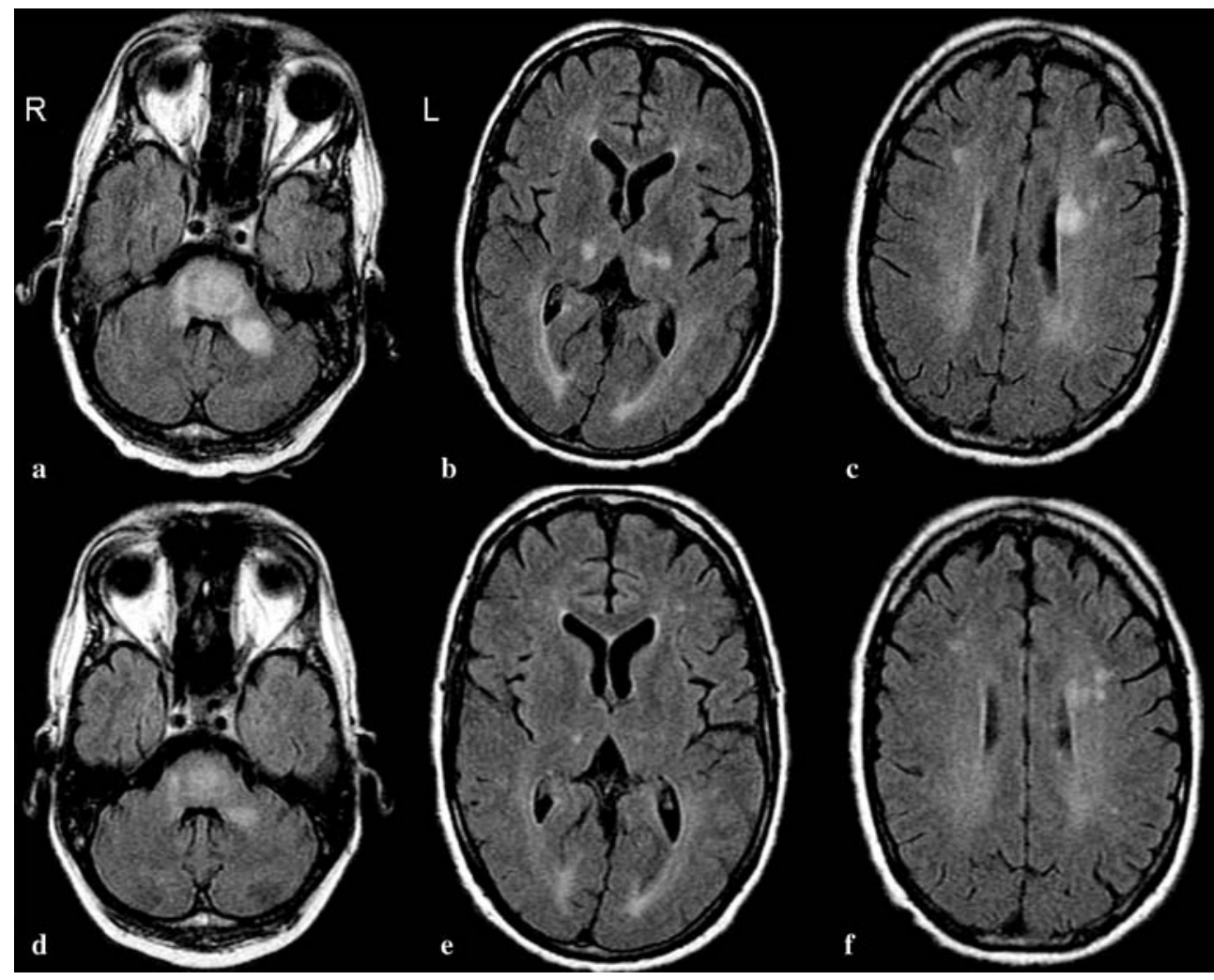

cerebral gadolinium-enhanced MRI was repeated, showing substantial amelioration of the intracerebral lesions (Fig. 1d-f). One year after treatment the patient still functions well, without relapse.

\section{Case discussion}

Elevated pressure, white cell count and protein level in the CSF in combination with the extensive white matter lesions confirmed the diagnosis of ADEM. ADEM is a rare cause of prolonged coma with complete recovery under specific treatment, and intensivists should be aware of this disease. Withdrawal or withholding of care in patients with prolonged coma can hardly be discussed as long as the cause of the coma remains unknown and the prognosis cannot be accurately established. Treatment with intravenous corticosteroids resulted in a full recovery. The absence of a previous infection is a peculiar aspect of our case.

\section{Comments}

This case illustrates the remarkable outcome after a long period of coma. Good recovery from a coma is observed in only $10 \%$ of reported cases and depends on the aetiology, the accompanying clinical signs, and the depth and duration of the coma [1]. ADEM is a rare monophasic illness that is thought to develop from antigenic mimicry, with antibodies having cross-reactivity to host epitopes in the nervous system. The disorder typically occurs following vaccination or a viral prodrome and is predominantly seen in children and in Japan, where viral encephalitis is more common [2, $3,4,5]$. A preceding infection is reported in $50-75 \%$ of cases $[3,4$, 5]. The estimated incidence is 0.8 per 100,000 population per year [3]. Some cases of ADEM among adults and the elderly have been reported, but the incidence is estimated to be considerably lower. The diagnosis of ADEM is reached on clinical grounds, evidence of white matter lesions on MRI and exclusion of other causes. These classic features were present in our case. However, diagnosis of ADEM may be difficult. Diagnostic overlap with multiple sclerosis (MS) may lead to underestimation of the prevalence $[4,5]$. Some $0-33 \%$ of children and $35 \%$ of adults initially diagnosed with ADEM will eventually develop MS $[4,5,6]$. Therefore this diagnosis should be considered, especially in adults. Follow-up by repeated MRI, at intervals not shorter than 6 months, may anticipate this development [7]. The differential diagnosis consists of meningo-encephalitis, meningitis and cerebral involvement in auto-immune diseases [8]. In severe cases, ADEM may lead to coma. There is no standard treatment for ADEM. It is a rare disease, and no formal clinical trials of any therapeutic agent have been published. Thus the management of the disease rests on strategies that have appropriate effects on the plausible disease mechanisms. Present treatments rely on immunosuppression and immunomodulation. 
Intravenous corticosteroids (preferably methylprednisolone) is the first choice of treatment and usually leads to full recovery. Plasma exchange and intravenous immunoglobulin should be considered by deterioration on corticosteroids $[9,10]$.

In conclusion, treatment of coma should be continued when the cause of the coma is unclear, even if there are numerous lesions in the brain. ADEM should be considered in comatose patients with raised CSF pressure, protein and lymphocytes, negative CSF cultures, and multiple white matter lesions on cerebral MRI. ADEM is predominantly seen in children, but also occurs in adults. Recovery may last weeks.

\section{References}

1. Bates D (2001) The prognosis of medical coma. J Neurol Neurosurg Psychiatry 71:20-23

2. Garg RK (2003) Acute disseminated encephalomyelitis. Postgrad Med J 79:11-17

3. Menge T, Hemmer B, Nessler F, Wiendl H, Neuhaus O, Hartung HP, Kieseier BC, Stuve O (2005) Acute disseminated encephalomyelitis: an update. Arch Neurol 62:1673-1680
4. Schwarz S, Mohr A, Knauth M, Wildemann B, Storch-Hagenlocher B (2001) Acute disseminated encephalomyelitis: a follow-up study of 40 adult patients. Neurology 56:1313-1318

5. Lin CH, Jeng JS, Hsieh ST, Yip PK, Wu RM (2007) Acute disseminated encephalomyelitis: a follow-up study in Taiwan. J Neurol Neurosurg Psychiatry 78:162-167

6. Wingerchuck DM (2006) The clinical course of acute disseminated encephalomyelitis. Neurol Res 28:341-347

7. Kesselring J, Miller DH, Robb SA, Kendall BE, Moseley IF, Kingsley D, du Boualy EP, McDonald WI (1990) Acute disseminated encephalomyelitis: MRI findings and the distinction from multiple sclerosis. Brain 113:291

8. Kennedy PGE (2004) Viral encephalitis: causes, differential diagnosis, and management. J Neurol Neurosurg Psychiatry 75:i10-i15

9. Bennetto L, Scolding N (2004) Inflammatory / post-infectious encephalomyelitis. J Neurol Neurosurg Psychiatry 75:i22-i28

10. Sahlas DJ, Miller SP, Guerin M, Veilleux M, Francis G (2000) Treatment of acute disseminated encephalomyelitis with intravenous immunoglobulin. Neurology 54:1370-1372

\section{J. M. P. Rovers (}

St. Elisabeth Hospital, Department of Neurology,

Hilvarenbeekseweg 60, Postbus 90151, 5000 LC Tilburg, The Netherlands e-mail: jmprovers@ hotmail.com

Tel.: +31-13-5391313

Fax: +31-13-5350355

\section{E.S. Louwerse} Jeroen Bosch Hospital, Department of Neurology,

Tolbrugstraat 11, Postbus 90153 ,

$5200 \mathrm{ME}$ 's Hertogenbosch,

The Netherlands

\section{C.P.C. de Jager}

Jeroen Bosch Hospital, Department of Intensive Care and Emergency Medicine, Tolbrugstraat 11, Postbus 90153, $5200 \mathrm{ME}$ 's Hertogenbosch,

The Netherlands 